\title{
Polarized photoluminescence from single wurtzite InP/InAs/InP core-multishell nanowires
}

\author{
Yasuaki Masumoto, ${ }^{1, a)}$ Yuuki Hirata, ${ }^{1}$ Premila Mohan, ${ }^{2}$ Junichi Motohisa, ${ }^{2}$ and \\ Takashi Fukui ${ }^{2}$ \\ ${ }^{1}$ Institute of Physics, University of Tsukuba, Tsukuba 305-8571, Japan \\ ${ }^{2}$ Research Center for Integrated Quantum Electronics, Hokkaido University, Sapporo 060-8628, Japan
}

(Received 1 April 2011; accepted 28 April 2011; published online 23 May 2011)

The photoluminescence from single InP/InAs/InP core-multishell nanowires (CMNs) was highly linearly polarized in perpendicular to the nanowire long axis. Taking account of dielectric confinement of light field, we found the internal optical dipole squared perpendicular to the nanotube long axis $d_{0 x}^{2}$ is larger than that parallel to the axis $d_{0 z}^{2}$ by two orders of magnitude. With the increase in the thickness of InAs nanotubes, the ratio $\beta=d_{0 x}^{2} / d_{0 z}^{2}$ increases. This strong anisotropy in $\beta$ comes from nearly perfect wurtzite structure of InP/InAs/InP CMNs and small inclusion of $p_{z}$-character into the $\Gamma_{7}(\mathrm{~B})$-hole state. (0) 2011 American Institute of Physics.

[doi:10.1063/1.3592855]

Semiconductor nanowires and nanotubes attract much attention recently because of the potential application to optical as well as electronic devices. InP/InAs/InP coremultishell nanowires (CMNs) are unique nanowires containing InAs hexagonal nanotubes. Nanotubes work as quantum wells and quantum wires and emit bright photoluminescence (PL) in the near infrared region. ${ }^{1}$ Another unique feature of $\mathrm{InP} / \mathrm{InAs} / \mathrm{InP}$ CMNs is the wurtzite structure instead of zincblende structure for both InAs and InP bulk crystals. ${ }^{2,3}$ The crystal field as well as spin-orbit interaction in the wurtzite structure split the top valence band into $\Gamma_{9}(\mathrm{~A})$-state, $\Gamma_{7}(\mathrm{~B})$-state, and $\Gamma_{7}(\mathrm{C})$-state. Optical transition between the valence $\Gamma_{9}(\mathrm{~A})$-state and the lowest conduction $\Gamma_{7}$-state is allowed only for the light polarization perpendicular to [0001] ( $c$-axis) while that between the valence $\Gamma_{7}(\mathrm{~B})$ - and $\Gamma_{7}(\mathrm{C})$-states and the lowest conduction $\Gamma_{7}$-state is allowed for both the light polarizations parallel and perpendicular to [0001]. So far, linear polarization of PL of wurtzite and zincblende InP, ${ }^{4}$ wurtzite $\mathrm{CdSe},{ }^{5}$ and GaN (Ref. 6) nanowires has been studied. PL of wurtzite InP nanowires is linearly polarized in perpendicular to the long axis of nanowires, [0001] (Ref. 4) while PL of wurtzite CdSe and wurtzite GaN nanowires is linearly polarized in parallel to [0001]. ${ }^{5,6}$ Understanding of polarized PL from wurtzite nanotubes and nanowires is at the elementary stage and is needed.

Optical absorption and PL of semiconductor nanowires strongly depend on the light polarization because large dielectric constant of nanowires suppresses internal electric field normal to the long axis of wires connecting with the external electric field. In a case of PL, cylindrical long nanowires $2 r$ in diameter having the dielectric constant of $\epsilon$ at the angular frequency of $\omega$, emitting dipole moment $d_{0}$ $=\left(d_{0 x}, d_{0 y}, d_{0 z}\right)$ in the nanowires can be replaced by the effective emitting dipole moment $d=\left(d_{x}, d_{y}, d_{z}\right)$ in the free space as follows: ${ }^{7}$

${ }^{a)}$ Electronic mail: shoichi@sakura.cc.tsukuba.ac.jp.

$$
\begin{aligned}
d_{x(y)} & \equiv A_{x(y)} d_{0 x(y)} \\
& =d_{0 x(y)} \sqrt{\epsilon} \frac{J_{1}^{\prime}(k r) H_{1}^{(1)}(k r)-J_{1}(k r) H_{1}^{(1) \prime}(k r)}{\sqrt{\epsilon_{0}} J_{1}^{\prime}(k r) H_{1}^{(1)}\left(k_{0} r\right)-\sqrt{\epsilon} J_{1}(k r) H_{1}^{(1) \prime}\left(k_{0} r\right)}, \\
d_{z} & \equiv A_{z} d_{0 z} \\
& =d_{0 z} \sqrt{\epsilon} \frac{J_{1}(k r) H_{0}^{(1)}(k r)-J_{0}(k r) H_{1}^{(1)}(k r)}{\sqrt{\epsilon} J_{1}(k r) H_{0}^{(1)}\left(k_{0} r\right)-\sqrt{\epsilon_{0}} J_{0}(k r) H_{1}^{(1)}\left(k_{0} r\right)},
\end{aligned}
$$

where $x(y)$-axis and $z$-axis are taken in perpendicular and in parallel to the long axis of the nanowires, respectively, $J_{m}$ and $H_{m}^{(1)}$ are the Bessel and first-kind Hankel functions, $k$ $=\sqrt{\epsilon} \omega / c$ and $k_{0}=\sqrt{\epsilon_{0}} \omega / c$ are wave numbers, $\epsilon_{0}$ is the static dielectric constant, and $c$ is the light velocity in vacuum. Then a ratio of $z$ linearly polarized PL intensity $I_{\|}$to $x(y)$ linearly polarized PL intensity $I_{\perp}$ is given by $I_{\|} / I_{\perp}=\left(2 d_{z}^{2}\right.$ $\left.+d_{x}^{2}\right) / 3 d_{x_{8}}^{2}$ and hence its degree of linear polarization $\rho$ is given by ${ }^{8}$

$$
\rho=\frac{I_{\|}-I_{\perp}}{I_{\|}+I_{\perp}}=\frac{d_{z}^{2}-d_{x}^{2}}{d_{z}^{2}+2 d_{x}^{2}}=\frac{A_{z}^{2}-A_{x}^{2}\left(d_{0 x}^{2} / d_{0 z}^{2}\right)}{A_{z}^{2}+2 A_{x}^{2}\left(d_{0 x}^{2} / d_{0 z}^{2}\right)} .
$$

Thus the ratio of internal dipole moments squared $d_{0 x}^{2} / d_{0 z}^{2} \equiv \beta$ is obtained by Eq. (2) from the measured $\rho$. As is numerically shown in the Ref. 6 , the conversion strongly depends on $\omega r / c$. In the limits of $\beta=0$ and $\infty, \rho$ approaches 1 and -0.5 , respectively.

A sample we studied is single InP/InAs/InP CMNs. An InP/InAs/InP CMN has hexagonal symmetry and contains an ultrathin wurtzite InAs hexagonal tube a few monolayers (MLs) thick surrounded by an inner thick wurtzite InP hexagonal pillar (core) $70 \mathrm{~nm}$ in diagonal length and an outer wurtzite InP hexagonal tube (shell) $35 \mathrm{~nm}$ thick. ${ }^{9}$ The CMNs have wurtzite structure and are elongated by $2 \mu \mathrm{m}$ toward [0001]. ${ }^{2}$ The hexagonal sidewalls of InP cores and InAs tubes have $\{1 \overline{1} 00\}$ faces ( $m$-planes). Lattice constants of free standing wurtzite InAs and InP are $a(\operatorname{InAs})=0.42 \mathrm{~nm}$, $c(\operatorname{InAs})=0.69 \mathrm{~nm}$ and $a(\operatorname{InP})=0.40 \mathrm{~nm}, c(\operatorname{InP})=0.66 \mathrm{~nm}$, respectively. ${ }^{2,3}$ Thickness of the InAs hexagonal tube, a few monolayers, is less than the critical thickness for the onset of 


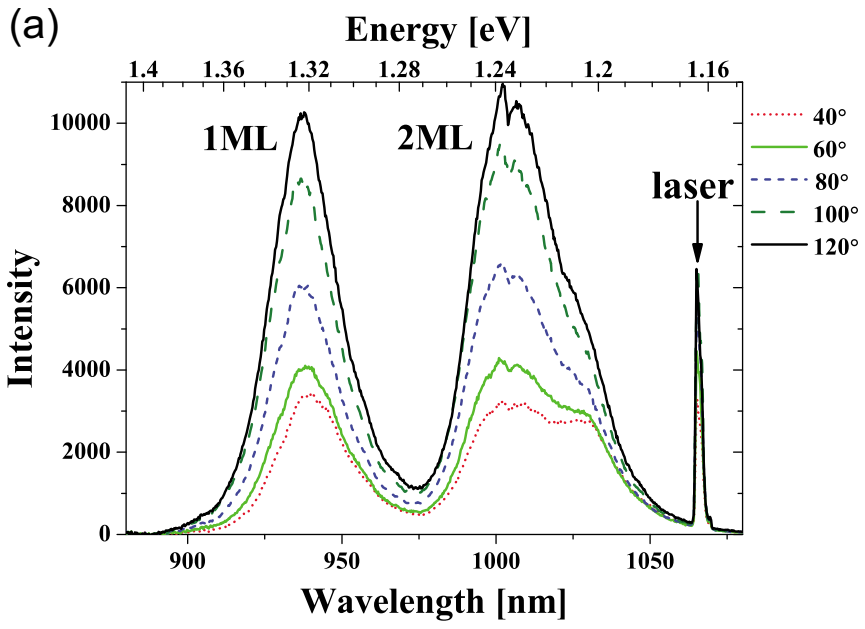

(b)
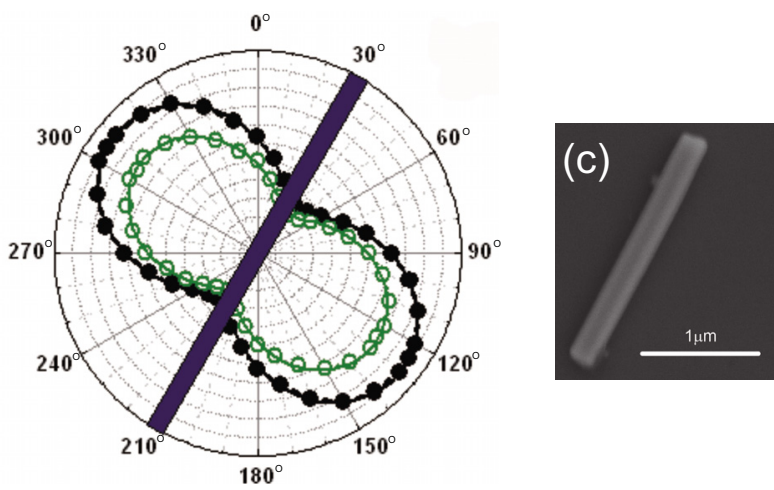

FIG. 1. (Color online) (a) Linearly polarized PL spectrum of an InP/InAs/ InP CMN. Laser denotes the second-order diffracted signal of the excitation laser at $532 \mathrm{~nm}$. (b) Azimuthal dependence of the integrated intensity of linearly polarized PL bands of the single InP/InAs/InP CMN. Solid circles correspond to the $1 \mathrm{ML}$ band and open circles to $2 \mathrm{ML}$ band. (c) Scanning electron microscope image of the single InP/InAs/InP CMN.

strain relaxation ${ }^{10}$ and therefore the ultrathin InAs hexagonal tube is considered to be coherently strained in all directions and to be in nearly hydrostatic strain field. Model-solid theory by Van de Walle explains that nearly hydrostatic strain field forms type-II band lineup. ${ }^{11,12}$ The PL spectrum of InP/ InAs/InP CMNs contains bands of type-II excitons consisting of holes in the InAs ultrathin hexagonal tube and electrons in the InP core or the InP outer shell. ${ }^{12}$ Large Stokes shift between PL and PL excitation (PLE) spectra ${ }^{12}$ and slow decay of PL with a decay tail lasting up to $300 \mathrm{~ns}$ indicate a type-II band lineup. Spectra of PL and PLE are composed of multiple bands due to ML scale variation in the InAs layer thickness. Multiple bands in macroscopic PL and PLE are much broader than the lifetime broadening and are inhomogeneously broadened. The spectra shown in Fig. 1(a) confirms that multiple bands and inhomogeneous broadening exist even in a single wire, as is reported previously. ${ }^{13}$

Single InP/InAs/InP CMNs lying on an InP substrate were found by means of an optical microscope and a scanning electron microscope. The sample was mounted on a cold finger of an optical cryostat and was cooled at $5 \mathrm{~K}$. Excitation laser wavelength was a $532 \mathrm{~nm}$ and the excitation density was as low as $3.45 \mu \mathrm{W} / \mu \mathrm{m}^{2}$. It generates electronhole pairs in an InP/InAs/InP CMN, holes flowed into the InAs nanotube and electrons in an InP core or clad and holes in InAs nanotubes recombined. Polarized PL from single InP/InAs/InP CMNs and bundles of a few parallel InP/InAs/

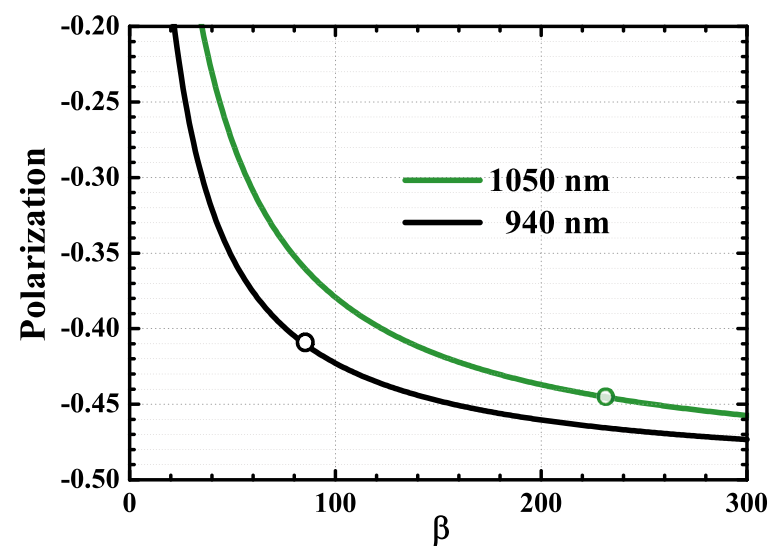

FIG. 2. (Color online) Degree of linear polarization of PL, $\rho$, as a function of the ratio of internal dipole moments squared $d_{0 x}^{2} / d_{0 z}^{2} \equiv \beta$ in the InP/InAs/ InP CMN for $1 \mathrm{ML}(940 \mathrm{~nm})$ and $2 \mathrm{ML}(1050 \mathrm{~nm})$ bands.

InP CMNs were measured by a liquid nitrogen cooled charge coupled device through an objective lenses of 0.42 numerical aperture with a magnification of 50, an infrared polarizer, a $20 \mathrm{~m}$ optical fiber, and a $25 \mathrm{~cm}$ monochromator. It was found the optical fiber completely randomizes the linear polarization of PL.

Figure 1(a) shows the linearly polarized PL spectra of an $\mathrm{InP} / \mathrm{InAs} / \mathrm{InP} \mathrm{CMN}$. Polarization direction is set by $\theta$, where $\theta$ is the angle between the polarization direction and an axis in the laboratory frame. Azimuthal dependence of the linearly polarized PL integrated intensity $I(\theta)$ is plotted in Fig. 1 (b) and is fitted by

$$
I(\theta)=P \cos ^{2}\left(\theta-\theta_{c}\right)+Q,
$$

where $P, Q$, and $\theta_{c}$ are positive fitting parameters. Because $I(\theta)$ reaches the maximum for the polarization perpendicular to the long axis of the nanowire, $\rho$ is negative and is given by $-P /(P+2 Q)$. The linear polarization $\rho=-0.48$ for $1 \mathrm{ML}$ PL and $\rho=-0.47$ for 2 ML PL. Measuring other nanowires, we note absolute value of $\rho=-0.44 \pm 0.04$ for 2 ML PL is systematically larger than that $\rho=-0.41 \pm 0.09$ for 1 ML PL. We also note $\rho=-0.41$ and $\rho=-0.44$ are very close to the minimum value of $\rho=-0.5$ corresponding to $\beta=\infty$.

We assume the conversion equation for the long cylinder $2 r$ in diameter shown in Eq. (2) is applied to the hexagonal nanowires $2 r=140 \mathrm{~nm}$ in diagonal length for simplicity. The ratio $d_{0 x}^{2} / d_{0 z}^{2} \equiv \beta$ in the InAs nanotube is derived from the measured $\rho$ by Eq. (2). Conversion equations, Eq. (2), are calculated for $1 \mathrm{ML}(940 \mathrm{~nm})$ and $2 \mathrm{ML}(1050 \mathrm{~nm})$ bands, as are shown in Fig. 2. Here $\epsilon=11.4$ and $\epsilon=10.8$ of InP are used for $940 \mathrm{~nm}$ and $1050 \mathrm{~nm},{ }^{14}$ respectively. The derived $\beta$ in the InAs nanotube for $1 \mathrm{ML}$ and $2 \mathrm{ML}$ bands are 85 and 230, respectively. ${ }^{15}$

The PL comes from type-II recombination between $s$-electrons in InP layers and $p$-holes in InAs layers. The $p$-holes in wurtzite InAs layers determines the ratio $\beta$ of the InAs nanotube.

On the quasicubic model, ${ }^{16-18}$ the topmost $\Gamma_{9}(\mathrm{~A})$-hole has the energy $E_{1}=\Delta_{\mathrm{cr}}+\Delta_{\mathrm{so}} / 3$ and the wave function in the form $\left|-\left(p_{x}+i p_{y}\right) / \sqrt{2} \uparrow\right\rangle$ or $\left|\left(p_{x}-i p_{y}\right) / \sqrt{2} \downarrow\right\rangle$ with which conduction $s$-electron in InP layers recombines emitting purely polarized light perpendicular to [0001], where $\Delta_{\text {cr }}$ and $\Delta_{\text {so }}$ are crystal field splitting and spin-orbit interaction, respectively. The second (third) highest valence $\Gamma_{7}(\mathrm{~B}[\mathrm{C}])$-hole has 
the energy $E_{2[3]}=\Delta_{\mathrm{cr}} / 2-\Delta_{\mathrm{so}} / 6 \pm \sqrt{\left(\Delta_{\mathrm{cr}}+\Delta_{\mathrm{so}}\right)^{2} / 4-2 \Delta_{\mathrm{cr}} \Delta_{\mathrm{so}} / 3}$ and the wave function in the form $a[b]\left|\left(p_{x}-i p_{y}\right) / \sqrt{2} \uparrow\right\rangle$ $+b[-a]\left|p_{z} \downarrow\right\rangle$ or $b[-a]\left|p_{z} \uparrow\right\rangle+a[b]\left|-\left(p_{x}+i p_{y}\right) / \sqrt{2} \downarrow\right\rangle$ in which conduction $s$-electron in InP layers recombines emitting polarized light parallel and perpendicular to [0001]. Thus the energy splittings between $\Gamma_{9}(\mathrm{~A})$ and $\Gamma_{7}[\mathrm{~B}(\mathrm{C})]$ holes are given by $\left(\Delta_{\mathrm{cr}}+\Delta_{\mathrm{so}}\right) / 2 \mp \sqrt{\left(\Delta_{\mathrm{cr}}+\Delta_{\mathrm{so}}\right)^{2} / 4-2 \Delta_{\mathrm{cr}} \Delta_{\mathrm{so}} / 3}$. Recent band calculation of wurtzite InAs shows energy splitting between $\Gamma_{9}(\mathrm{~A})$-hole and $\Gamma_{7}[\mathrm{~B}(\mathrm{C})]$-hole is $0.105 \mathrm{eV}(0.469$ eV). ${ }^{19}$ Equating two calculated energy splittings to the expression above gives $\Delta_{\mathrm{cr}}=0.379 \mathrm{eV}$ and $\Delta_{\mathrm{so}}=0.195 \mathrm{eV}{ }^{19}$ Polarized PL intensities $I_{\|}$and $I_{\perp}$ are proportional to $b^{2}=2 \Delta_{\mathrm{so}}^{2} / 9\left(E_{2}^{2}+2 \Delta_{\mathrm{so}}^{2} / 9\right) \quad$ and $a^{2}=E_{2}^{2} /\left(E_{2}^{2}+2 \Delta_{\mathrm{so}}^{2} / 9\right)$, respectively. ${ }^{18}$ By using $\Delta_{\text {so }}=0.195 \mathrm{eV}$ and $\Delta_{\mathrm{cr}}=0.379 \mathrm{eV}$, we obtain $b^{2}=0.07$ and $a^{2}=0.93$ are obtained. The ratio $\beta$ for the transition between $\Gamma_{7}(\mathrm{~B})$-hole and conduction $s$-electron in InP layers is 14 and that for the transition between $\Gamma_{7}(\mathrm{C})$-hole and conduction $s$-electron in InP layers is 0.07 .

In quantum wells in InAs nanotubes, the well depth for $\Gamma_{9}(\mathrm{~A})$-hole is considered to be $0.32 \mathrm{eV}$ (Ref. 12) and that for $\Gamma_{7}(\mathrm{~B})$-hole $0.215 \mathrm{eV}$. There is only one quantized hole state in 1 and $2 \mathrm{ML}$ wells and their energies approach to each other for the ultrathin quantum wells, because effective mass of $\Gamma_{9}(\mathrm{~A})$-hole perpendicular to [0001], $m_{\mathrm{A} \perp}=0.084 m_{0}$ is smaller than effective mass of $\Gamma_{7}(\mathrm{~B})$-hole perpendicular to [0001], $m_{\mathrm{B} \perp}=0.113 m_{0} .{ }^{19}$ This energy approach induces the mixing of $\Gamma_{9}(\mathrm{~A})$-hole and $\Gamma_{7}(\mathrm{~B})$-hole and hence brings forth finite $\beta$. With the decrease in the well thickness from 2 to 1 ML, the mixing of $\Gamma_{9}(\mathrm{~A})$ and $\Gamma_{7}(\mathrm{~B})$-holes increases in the ultrathin InAs quantum wells in consistent with the observed decrease in $\beta$. Very large $\beta$ comes from nearly perfect wurtzite structure of InP/InAs/InP CMNs and small inclusion of $p_{z}$-character into the $\Gamma_{7}(\mathrm{~B})$-hole state in wurtzite InAs. We believe density of stacking faults in InP/InAs/InP CMNs studied in this letter is low. $2,3,20$

In summary, we observed the PL from single InP/InAs/ InP CMNs was highly linearly polarized in perpendicular to the long axis of the nanowire. Taking account of dielectric confinement of light field, we found the optical dipole squared perpendicular to the nanotube long axis $d_{x}^{2}$ is larger than that parallel to the axis $d_{z}^{2}$ by two orders of magnitude. With the increase in the thickness of InAs nanotubes, the ratio $\beta=d_{x}^{2} / d_{z}^{2}$ increases. This strong anisotropy in the oscillator strength comes from nearly perfect wurtzite structure of InP/InAs/InP CMNs and small inclusion of $p_{z}$-character into the $\Gamma_{7}(\mathrm{~B})$-hole state.

This work was supported by Grant-in-Aid for Scientific Research No. 20244044 from the MEXT of Japan.

${ }^{1}$ Y. Masumoto, K. Goto, S. Yoshida, Y. Sakuma, P. Mohan, J. Motohisa, and T. Fukui, Phys. Rev. B 82, 075313 (2010).

${ }^{2}$ P. Mohan, J. Motohisa, and T. Fukui, Appl. Phys. Lett. 88, 013110 (2006).

${ }^{3}$ P. Mohan, J. Motohisa, and T. Fukui, Nanotechnology 16, 2903 (2005).

${ }^{4}$ A. Mishra, L. V. Titova, T. B. Hoang, H. E. Jackson, L. M. Smith, J. M. Yarrison-Rice, Y. Kim, H. J. Joyce, Q. Gao, H. H. Tan, and C. Jagadish, Appl. Phys. Lett. 91, 263104 (2007).

${ }^{5}$ C. X. Shan, Z. Liu, and S. K. Hark, Phys. Rev. B 74, 153402 (2006).

${ }^{6}$ H.-Y. Chen, Y.-C. Yang, H.-W. Lin, S.-C. Chang, and S. Gwo, Opt. Express 16, 13465 (2008).

${ }^{7}$ H. E. Ruda and A. Shik, J. Appl. Phys. 100, 024314 (2006).

${ }^{8}$ H. E. Ruda and A. Shik, Phys. Rev. B 72, 115308 (2005).

${ }^{9}$ P. Mohan, J. Motohisa, and T. Fukui, Appl. Phys. Lett. 88, 133105 (2006).

${ }^{10}$ J. Cibert, Y. Gobil, L. S. Dang, S. Tatarenko, G. Feuillet, P.H. Jouneau, and K. Saminadayar, Appl. Phys. Lett. 56, 292 (1990).

${ }^{11}$ C. G. Van de Walle, Phys. Rev. B 39, 1871 (1989).

${ }^{12}$ B. Pal, K. Goto, M. Ikezawa, Y. Masumoto, P. Mohan, J. Motohisa, and T. Fukui, Appl. Phys. Lett. 93, 073105 (2008).

${ }^{13}$ K. Goto, M. Ikezawa, S. Tomimoto, B. Pal, Y. Masumoto, P. Mohan, J. Motohisa, and T. Fukui, Jpn. J. Appl. Phys., Part 1 48, 04C203 (2009).

${ }^{14}$ Handbook of Optics, Devices, Measurements, and Properties, 2nd ed., (McGraw-Hill, New York, 1994), Vol. 2.

${ }^{15}$ If we assume the conversion equation for the long cylinder $1.82 r$ in diameter shown in Eq. (2) is applied to the hexagonal nanowires $2 r$ in diagonal length for the conservation of cross-sectional area, the derived $\beta$ in the InAs nanotube for $1 \mathrm{ML}$ and $2 \mathrm{ML}$ bands are 125 and 305, respectively.

${ }^{16}$ J. J. Hopfield, J. Phys. Chem. Solids 15, 97 (1960).

${ }^{17}$ G. L. Bir and G. E. Pikus, Symmetry and Strain-Induced Effects in Semiconductors (Wiley, New York, 1974).

${ }^{18}$ S. L. Chuang and C. S. Chang, Phys. Rev. B 54, 2491 (1996).

${ }^{19}$ A. De and C. E. Pryor, Phys. Rev. B 81, 155210 (2010).

${ }^{20}$ Y. Kitauchi, Y. Kobayashi, K. Tomioka, S. Hara, K. Hiruma, T. Fukui, and J. Motohisa, Nano Lett. 10, 1699 (2010). 